Helbig, Marko; Grießbach, Gert; Witte, Herbert; Schack, Bärbel:

\title{
Numerisch stabile Normierung des indirekt geschätzten Bispektrums
}

\author{
Zuerst erschienen in: $\quad$ Biomedizinische Technik = Biomedical Engineering. - Berlin [u.a.] : \\ de Gruyter. - 46 (2001), S1, S. 318-319. \\ Jahrestagung der Deutschen Gesellschaft für Biomedizinische \\ Technik (DGBM) im VDE ; 35 (Bochum) : 2001.09.19-21 \\ Erstveröffentlichung: 2001 \\ Datum Digitalisierung: $\quad$ 2009-08-06 \\ ISSN (online): $\quad$ 1862-278X \\ ISSN(print) $\quad$ 0013-5585 \\ DOI: $\quad$ 10.1515/bmte.2001.46.s1.318 \\ [Zuletzt gesehen: 2019-12-06]
}

„Im Rahmen der hochschulweiten Open-Access-Strategie für die Zweitveröffentlichung identifiziert durch die Universitätsbibliothek IImenau."

"Within the academic Open Access Strategy identified for deposition by IImenau University Library."

„Dieser Beitrag ist mit Zustimmung des Rechteinhabers aufgrund einer (DFGgeförderten) Allianz- bzw. Nationallizenz frei zugänglich."

„This publication is with permission of the rights owner freely accessible due to an Alliance licence and a national licence (funded by the DFG, German

Research Foundation) respectively."

\section{DFG}

Nationallizenzen 


\title{
NUMERISCH STABILE NORMIERUNG DES INDIREKT GESCHÄTZTEN BISPEKTRUMS
}

\author{
M. Ilclbig', G. Gricßbach', H. Witte², B. Schack ${ }^{2}$ \\ 'Institut f. Biomed. Technik und Med. Informatik, TU Ilmenau, Deutschland \\ ${ }^{2}$ Institut f. Med. Statistik, Informatik und Dokumentation, FSU Jena, Deutschland
}

Marko.Helbig@informatik.tu-ilmenau.de

\section{EINLEITUNG}

Klassische Ansătze der Bispektralanalyse sind auf ein festes Analyseintervall bezogen und setzen $u$. a. Stationarităt der zu untersuchenden Signale voraus. Da Biosignale diesen Anforderungen zumeist nicht genügen, wurde cine neue Methode zur zeitvarianten Bispektralanalyse auf der Basis einer adaptiv rekursiven Schätzung der Kumulanten III. Ordnung entwickelt und in [1] vorgestellt.

Eine Quantifizierung der Stärke quadratischer Phasenkopplungen setzt allerdings Signalamplitudenunabhängigkeit voraus. Auf der Basis des direkten Zuganges (Periodogramm III. Ordnung) sind Bikohärenzschätzungen seit langem bekannt [2, 3, 4]. Die Normierungsverfahren des direkt geschätzten Bispektrums führen aber zu numerischen Problemen beim indirekt geschätzten Bispektrum, die eine Interpretation der Bikohärenzmatrix stellenweise unmöglich machen. Da der dynamische Ansatz [1] auf der indirekten Schätzung beruht, wird ein geeignetes Normierungsverfahren entwickelt und vorgestellt.

\section{METHODEN}

Das Bispektrum $B\left(\omega_{1}, \omega_{2}\right)$ der stationären Zeitreihe $\left\{x_{k}\right\}_{k=0,1, \ldots}$ ist definiert als dic Fouriertransformation der Kumulante III. Ordnung.

$$
B\left(\omega_{1}, \omega_{2}\right)=\sum_{\tau_{1}=-\infty}^{\infty} \sum_{\tau_{2}=-\infty}^{\infty} c_{3}\left(\tau_{1}, \tau_{2}\right) \cdot \exp \left\{-i \cdot\left(\omega_{1} \tau_{1}+\omega_{2} \tau_{2}\right)\right\}
$$

Zur Schätzung dieser Größe wird das zu untersuchende Signal in $\mathbf{N}$ Epochen unterteilt. Der direkte Ansatz beinhaltet die Momentenbildung der Fouriertransformierten dieser Signalepochen (2), der indirekte Ansatz erfolgt über die Fouriertransformation der empirischen Kumulante III. Ordnung (3).

$$
\begin{gathered}
B^{i}\left(\omega_{1}, \omega_{2}\right)=X^{i}\left(\omega_{1}\right) \cdot X^{i}\left(\omega_{2}\right) \cdot\left(X^{i}\right)^{i}\left(\omega_{1}+\omega_{2}\right) \\
B^{i}\left(\omega_{1}, \omega_{2}\right)=\sum_{m=-L}^{L} \sum_{n=-L}^{L} r_{3}^{i}(m, n) \cdot \exp \left\{-i \cdot\left(\omega_{1} m+\omega_{2} n\right)\right\}
\end{gathered}
$$

Durch Mittelung dieser Ausdrücke gelangt man zu einer konsistenten Schätzung des Bispektrums:

$$
\hat{B}\left(\omega_{1}, \omega_{2}\right)=\frac{1}{N} \sum_{i=1}^{N} B^{i}\left(\omega_{1}, \omega_{2}\right)=\left\langle B^{i}\left(\omega_{1}, \omega_{2}\right)\right\rangle
$$

Um eine sachgerechte Quantifizierung quadratischer Phasenkopplungen zu ermöglichen, muß der Einfluß der Signalamplitude eliminiert und deshalb das Bispektrum normiert werden (Bikohärenz).

Von Haubrich [3] wurde eine Bikohärenzschätzung (5) eingeführt, die sich direkt aus der Definition der Kohärenz n-ter Ordnung [2, S. 31] ergibt.

$$
\hat{b}_{\text {direct }}^{1}\left(\omega_{1}, \omega_{2}\right)=\frac{\left|\left\langle B^{i}\left(\omega_{1}, \omega_{2}\right)\right\rangle\right|}{\sqrt{\left\langle\left. X^{i}\left(\omega_{1}\right)\right|^{2}\right\rangle \cdot\left\langle\left|X^{i}\left(\omega_{2}\right)\right|^{2}\right\rangle \cdot\left\langle\left|X^{i}\left(\omega_{1}+\omega_{2}\right)\right|^{2}\right\rangle}}
$$

Da diese Form nicht auf den Wertebereich zwischen 0 und 1 beschränkt ist, wurde von Kim u. Powers [4] eine zweite Form entwickelt (6), die im Falle der direkten Bispektrumschätzung eine Korrelation zwischen $X^{i}\left(\omega_{1}\right) \cdot X^{i}\left(\omega_{2}\right)$ und $X^{i}\left(\omega_{1}+\omega_{2}\right)$ darstellt und somit zwischen 0 und 1 begrenzt ist.

$$
\hat{b}_{\text {direct }}^{\text {II }}\left(\omega_{1}, \omega_{2}\right)=\frac{\left|\left\langle B^{i}\left(\omega_{1}, \omega_{2}\right)\right\rangle\right|}{\sqrt{\left\langle\left|X^{i}\left(\omega_{1}\right) \cdot X^{i}\left(\omega_{2}\right)\right|^{2}\right\rangle \cdot\left\langle\left|X^{i}\left(\omega_{1}+\omega_{2}\right)\right|^{2}\right\rangle}}
$$

Die Anwendung dieser Normierung auf das indirekt geschätzte Bispektrum führt zur folgenden Gleichung,

$$
\hat{\mathrm{b}}_{\text {indirect }}^{\mathrm{II}}\left(\omega_{1}, \omega_{2}\right)=\frac{\left|\left\langle\mathrm{B}^{i}\left(\omega_{1}, \omega_{2}\right)\right\rangle\right|}{\sqrt{\left\langle\mathrm{P}^{i}\left(\omega_{1}\right) \cdot \mathrm{P}^{i}\left(\omega_{2}\right)\right\rangle \cdot\left\langle\mathrm{P}^{i}\left(\omega_{1}+\omega_{2}\right)\right\rangle}}
$$

wobei $\mathrm{P}^{i}(\omega)$ das Leistungsspektrum der i-ten Epoche darstellt, das sich aus der Fouriertransformation der empirischen Kumulante II. Ordnung ergibt.

$$
P^{i}(\omega)=\sum_{m=-L}^{L} r_{2}^{i}(m) \cdot \exp \{-i \omega\}
$$

Es zeigte sich, daß diese Bikohärenzschätzung zu enormen numerischen Problemen führt, die eine 
Interpretation der resultierenden Bikohärenzmatrix unmöglich machen. Besonders an Frequenzen, an denen (im Bispektrum) nur verschwindend kleine Leistungen vorhanden sind, ergibt die Schätzung nach (7) Werte, die weit über Eins hinausgehen. Die Ursache der numerischen Effekte liegt in der Quotientenbildung unterschiedlich konstruierter Terme (Kumulanten II. vs. Ill. Ordnung), die bei sehr kleinen Leistungswerten numerisch instabil wird. Im Gegensatz zu (6) stellt (7) keine unmittelbare Korrelation dar.

Zur Lösung dieser Probleme wurde ein drittes Normalisierungsverfahren entwickelt:

$$
\hat{b}^{\mathrm{ml}}\left(\omega_{1}, \omega_{2}\right)=\frac{\left|\left\langle\mathrm{B}^{\mathrm{i}}\left(\omega_{1}, \omega_{2}\right)\right\rangle\right|}{\sqrt{\left\langle\left.\mathrm{B}^{\mathrm{i}}\left(\omega_{1}, \omega_{2}\right)\right|^{2}\right\rangle}}
$$

Aufgrund der Dreiecks-Ungleichung

$$
\left|\left\langle B^{i}\left(\omega_{1}, \omega_{2}\right)\right\rangle\right| \leq\left\langle B^{i}\left(\omega_{1}, \omega_{2}\right) \mid\right\rangle
$$

und der Cauchy-Ungleichung

$$
\left\langle B^{i}\left(\omega_{1}, \omega_{2}\right) \mid\right\rangle \leq \sqrt{\left\langle\left|B^{i}\left(\omega_{1}, \omega_{2}\right)\right|^{2}\right\rangle}
$$

besitzt diese Bikohärenzschătzung einen Wertebereich zwischen 0 und 1.

\section{ERGEBNISSE}

Es wurden die statistischen Eigenschaften der neu entwickelten Normierung (9) anhand zweier Signalmodelle untersucht und mit den bekannten Schätzungen (5) und (6) verglichen.

- Stationärer Rauschprozeß:

In Analogie zur Arbeit von Elgar und Guza [6] wurden Gauß-verteilte Zufallsprozesse unterschiedlicher Länge mit einem Erwartungswert von Null und einer Varianz von Eins simuliert. Zur Analyse der Abhängigkeit der statistischen Parameter von N wurden für jedes N 100 Simulationen durchgeführt und die Ergebnisse gemittelt. In den folgenden Abbildungen werden die Verläufe der 95\%-Quantilwerte (Abb. 1) und der 99\%-Quantilwerte (Abb. 2) in Abhängigkeit von $\mathrm{N}$ dargestellt.

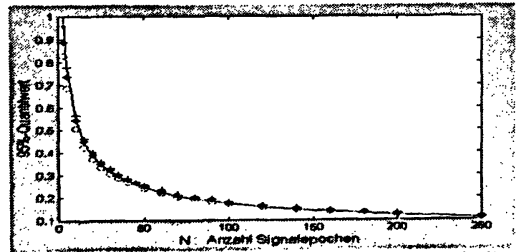

Abb. 1: $95 \%$-Quantilwerte (+ nach (5), * nach (6), o nach (9))

Es kann festgestellt werden, daß die Quantilwerte aller drei Schătzungen für steigendes $\mathrm{N}$ asymptotisch identisch sind. Die von Elyar und Guza [5] formulierten Signifikanzgrenzen (95\%: $\sqrt{3} / \sqrt{\mathrm{N}}$ und $99 \%$ : $\sqrt{4.6} / \sqrt{\mathrm{N}})$ können auch durch die neue Normierungsform bestätigt werden.

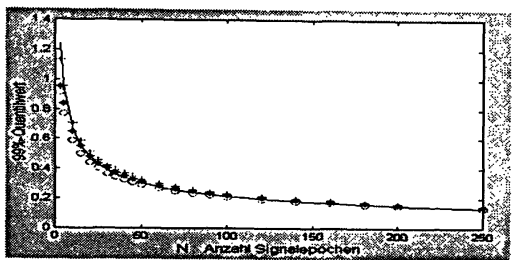

Abb. 2: 99\%-Quantilwerte (+ nach (5), * nach (6), o nach (9))

- Summation periodischer Schwingungen

Bei diesem Signalmodell wird der Grad einer quadratischen Phasenkopplung durch Phasenverrauschung variiert [6]. Hierbei liefern alle drei Schätzer sogar identische Ergebnisse. Die Gleichheit der Ergebnisse für diese Simulation läßt sich unmittelbar aus den Formeln (5), (6) und (9) ableiten.

Die Anwendung der Bikohärenzschätzung (9) zur Quantifizierung nichtlinearer Phasenkopplungen bei Herzfrequenzkomponenten führte zu den gleichen Ergebnissen wie die direkte Bikohärenzschätzung [7].

\section{ZUSAMMENFASSUNG}

In der vorliegenden Arbeit wurde eine neue Form zur numerisch stabilen Normierung des indirekt geschătzten Bispektrums vorgestellt und ihre statistischen Eigenschaften anhand von Simulationen untersucht. Sie bildet die entscheidende Grundlage zur Erweiterung der zeitvarianten Bispektralanalyse [1] zur zeitvarianten Bikohärenzanalyse.

\section{LITERATURHINWEISE}

[1] Helbig, M., Grießbach, G., Schack, B., Witte, H.: Application of time-variant bispectrum in biosignal analysis. EMBEC, Vienna (Austria), 1999, 392-393

[2] Nikias C.L., Petropulu A.P.: Higher-order spectra analysis. PTR Prentice Hall, Englewood Cliffs, 1993

[3] Haubrich, R.: Earth noise, 5 to 500 millicycls per second. J. Geophys. Res. vol.70, 1965, 1415-1427

[4] Kim, Y., Powers, E.: Digital bispectral analysis and its application to nonlinear wave interactions. IEEE Trans. Plasma Science, vol. PS-7, 1979, 120-131

[5] Elgar, S., Guza, R. T.: Statistics of bicoherence. IEEE Trans. on ASSP, vol. 36 (1988), 1667-1668

[6] Sebert, G., Elgar, S.: Statistics of bicoherence and biphase. Proc. Workshop Higher-Order Spectral Analysis, Vail CO, June 1989, 223-228

[7] Witte, H., Putsche, P., Eisclt, M.. Amold, M1.. Schmidt, K., Schack, B.: Technique for the Quantification of Transient Quadratic Phase Couplings between lleart Rate Components. Biomed. Techik 46 (2001), $42-49$

Diese Arbeit wurde gefordert durch dic DI:(i-Projehte GR 1555/2-3 und W' $1166 / 2-3$. 